\title{
Sandra Bohlinger
}

\section{Zur Wirksamkeit von Maßnahmen aktiver Arbeitsmarktpolitik}

\begin{abstract}
Der Beitrag geht der Frage nach der Wirksamkeit der Maßnahmen aktiver Arbeitsmarktpolitik (ALMP - active labour market policies) nach, die seit mittlerweile rund 20 Jahren als politische Strategie zur Reduktion von Arbeitslosigkeit eingesetzt werden. Im Zentrum steht dabei in Förderung beruflicher Bildung zur Erhöhung der Arbeitsmarktteilhabe. Ausgangspunkt bildet die Ausgabenentwicklung und -verteilung von ALMP in Deutschland und Europa. Daran anschließend werden die Probleme der Erfassung und des Nachweises der Wirksamkeit von ALMP skizziert und Befunde über die Impacts unterschiedlicher ALMP-Typen im deutschen und internationalen Kontext vorgestellt. Daraus lässt sich eine Reihe von Erkenntnissen ableiten, die als Voraussetzung für die Konzeption erfolgreicher ALMP gelten und Schlussfolgerungen für die Weiterentwicklung aktiver Arbeitsmarktpolitik zulassen.
\end{abstract}

\section{$1 \quad$ Einleitung ${ }^{1}$}

Um das Qualifikationsniveau von Individuen nachhaltig zu erhöhen, Arbeitslosigkeit zu reduzieren und mehr Menschen die Teilhabe am Arbeitsmarkt zu gewährleisten, werden in der Mehrheit der europäischen Staaten beschäftigungsfördernde Maßnahmen im Rahmen aktiver Arbeitsmarktpolitik (active labour market policies - ALMP) durchgeführt, deren Wirksamkeit aus ökonomischer, pädagogischer und soziologischer Perspektive allerdings kontrovers diskutiert wird.

Basis für die Diskussionen bilden die offiziellen nationalen Arbeitslosenstatistiken und die eher inkonsistenten Teilnahmestatistiken an den ALMP-Maßnahmen. Hierbei lassen sich zunächst drei verschiedene Typen von Maßnahmen differenzieren, nämlich

- solche, die durch die Förderung beruflicher Weiterbildung vor allem auf die (Wieder)Eingliederung in reguläre Beschäftigung zielen,

- solche, bei denen die Reintegration eher ein Nebenziel ist (wie bei Arbeitsbeschaffungsoder Strukturanpassungsmaßnahmen) sowie

- $\quad$ solche, die auf die Reduzierung des Arbeitsangebots zielen (z.B. Frühverrentung). Besonderes Interesse gilt allen Maßnahmen, die durch die Vermittlung beruflicher Bildung die Aufnahme regulärer Beschäftigung fördern sollen. Ihre Ziele liegen in der Anpassung an die sich wandelnden Herausforderungen des Arbeitsmarktes, der Erhöhung ökonomischer Effizienz, der Produktivität und damit der Vermeidung einer Wissensveraltung. Letztlich dient die Förderung beruflicher Bildung auch der Erhöhung der kulturellen und

1 Der Beitrag entstand im Rahmen eines Projektes zur beruflichen Weiterbildung älterer, an- und ungelernter Arbeitnehmer und Arbeitnehmerinnen in Kooperation mit dem Bildungswerk der Hessischen Wirtschaft und der Bundesagentur für Arbeit.

Arbeit, Heft 2, Jg. 16 (2007), S. 132-147 
gesellschaftlichen Teilhabe.

Bei den Ausgaben für ALMP und Sozialleistungen für Arbeitslosigkeit lässt sich in den europäischen Mitgliedstaaten kein einheitlicher Trend beobachten. Vielmehr ist die Entwicklung der Ausgaben stark länder- und politikabhängig. So ist deutlich zu erkennen, dass in allen neuen Mitgliedstaaten ${ }^{2}$ mit Ausnahme Polens die Ausgaben in den vergangenen zehn Jahren kontinuierlich gestiegen sind. Die Orientierung an der Europäischen Union, der Ausbau der Sozialsysteme und der Beginn regelmäßiger Datenerhebungen dürften bei diesen Entwicklungen eine entscheidende Rolle gespielt haben:

Tab. 1: Sozialausgaben für Arbeitslosigkeit 2004 in ausgewählten EULändern ${ }^{3}$

\begin{tabular}{|l|r|r|c|}
\hline Land & in Millionen Euro & $\begin{array}{c}\text { in \% der Gesamt- } \\
\text { ausgaben }\end{array}$ & in \% des BIP \\
\hline EU-25 & 178.406 & 6,5 & 1,7 \\
\hline EU-15 & 175.079 & 6,6 & 1,8 \\
\hline BE & 10.069 & 12,5 & 3,5 \\
\hline CZ & 646 & 3,9 & 0,7 \\
\hline DK & 5.564 & 9,5 & 2,8 \\
\hline DE & 53.759 & 8,6 & 2,4 \\
\hline ES & 21.027 & 12,9 & 2,5 \\
\hline FR & 37.813 & 7,8 & 2,3 \\
\hline IE & 1.986 & 8,3 & 1,3 \\
\hline IT & 6.913 & 2,0 & 0,5 \\
\hline LT & 37 & 1,6 & 0,2 \\
\hline HU & 492 & 2,9 & 0,6 \\
\hline MT & 56 & 6,9 & 1,3 \\
\hline NL & 8.162 & 6,3 & 1,7 \\
\hline AT & 3.977 & 6,0 & 1,7 \\
\hline PL & 1.378 & 3,5 & 0,7 \\
\hline PT & 1.887 & 5,7 & 1,3 \\
\hline SK & 349 & 6,2 & 1,0 \\
\hline FI & 3.847 & 9,8 & 2,5 \\
\hline SE & 5.551 & 6,2 & 2,0 \\
\hline UK & 11.728 & 2,6 & 0,7 \\
\hline & & & \\
\hline
\end{tabular}

Während in den meisten EU-15-Staaten eine Senkung der Ausgaben bis zur Jahrtausendwende mit einem anschließenden Ausgabenanstieg zu erkennen ist, haben nur wenige Länder wie Frankreich oder Belgien eine kontinuierliche Zunahme der Ausgaben in den 2 Beitrittsjahr 2004

3 Quelle: EUROSTAT (2007) 
vergangenen zehn Jahren zu verzeichnen. Dies trifft vor allem auf die Staaten zu, die ihre sozialen Sicherungssysteme trotz anhaltender Arbeitslosigkeit nicht grundlegend reformiert haben. Länder, die entsprechende Reformen ab Mitte der 1990er Jahre umgesetzt haben, weisen dagegen eine langfristige Senkung der Ausgaben auf, wobei es sich ausschließlich um die nordeuropäischen Staaten handelt.

Tendenziell lässt sich dabei auch erkennen, dass in Staaten mit hohem sozialem Schutz ${ }^{4}$ auch die Ausgaben für die Folgen von Arbeitslosigkeit überdurchschnittlich hoch sind. Lagen die Ausgaben in diesem Bereich 2004 in der EU-25 bei 6,5\% aller Sozialausgaben (EU-15: 6,6\%), so waren sie in dagegen in Belgien, Dänemark und Deutschland überdurchschnittlich hoch.

Deutschland hat in diesem Kontext rund 53 Milliarden Euro für die Abfederung der Folgen von Arbeitslosigkeit ausgegeben. ${ }^{5}$ Davon entfällt die Mehrheit auf die unterschiedlichen Formen von Arbeitslosengeld, während lediglich fünf Milliarden für aktive Maßnahmen aufgewendet werden, darunter drei Milliarden für Mobilitätshilfen zur Aufnahme einer Beschäftigung, zwei Milliarden für Maßnahmen der beruflichen Weiterbildung und 148 Millionen für Beratungstätigkeiten (EUROSTAT 2007, 174). Solche Maßnahmen können zudem danach differenziert werden, ob sie in erster Linie auf die Vermittlung praxisbezogener Kenntnisse, Fähigkeiten und Fertigkeiten ausgerichtet sind oder (zugleich) einen mehr oder minder erheblichen Theorieanteil einschließen. Die Ausgaben für die arbeitsmarktpolitischen Instrumente zur Förderung der Berufs- und Beschäftigungsfähigkeit verteilen sich dabei wie folgt:

4 Und vor allem mit hohem Kündigungsschutz

5 Diese Angabe gilt ebenfalls für 2004 
Tab. 2: Ausgewählte arbeitsmarktpolitische Instrumente in Deutschland (Berichtsjahr 2005) $^{6}$

\begin{tabular}{|l|c|c|}
\hline \multicolumn{1}{|c|}{ Merkmal } & $\begin{array}{c}\text { Jahresdurchschnitts- } \\
\text { bestand }\end{array}$ & $\begin{array}{c}\text { Jahressumme Zugang/ } \\
\text { Eintritte/ Bewilligungen }\end{array}$ \\
\hline $\begin{array}{l}\text { Beratung und Unterstüt- } \\
\text { zung der Arbeitssuche }\end{array}$ & $\mathbf{1 1 6 5 5 4}$ & $\mathbf{2 3 3 5 9 7 1}$ \\
\hline Qualifizierungsmaßnahmen & $\mathbf{2 2 2 ~ 4 6 1}$ & $\mathbf{1 0 5 9 2 0 5}$ \\
\hline Davon & 114350 & 131521 \\
\hline Berufliche Weiterbildung & 34021 & 33208 \\
\hline $\begin{array}{l}\text { Berufliche Wiederein- } \\
\text { gliederung Behinderter }\end{array}$ & 69000 & 894476 \\
\hline $\begin{array}{l}\text { Eignungsfeststellungs- und } \\
\text { Trainingsmaßnahmen }\end{array}$ & 5553 & 314756 \\
\hline Deutsch-Sprachlehrgänge ${ }^{1}$ & 406150 & 663595 \\
\hline $\begin{array}{l}\text { Berufsberatung und Förde- } \\
\text { rung der Berufsausbildung }\end{array}$ & 415479 & 398538 \\
\hline $\begin{array}{l}\text { Beschäftigungsbegleitende } \\
\text { Leistungen }\end{array}$ & & 265057 \\
\hline Davon & 93005 & 716063 \\
\hline $\begin{array}{l}\text { Förderung abhängiger } \\
\text { Beschäftigung }\end{array}$ & 322474 & 213085 \\
\hline $\begin{array}{l}\text { Förderung der Selbst- } \\
\text { ständigkeit }\end{array}$ & 287010 & 5302675 \\
\hline $\begin{array}{l}\text { Beschäftigung schaffende } \\
\text { Maßnahmen }\end{array}$ & 46588 & \\
\hline $\begin{array}{l}\text { Sonstiges (ESF-BA-Pro- } \\
\text { gramme, freie Förderung) }\end{array}$ & 1494242 & \\
\hline Summe & & \\
\hline $\begin{array}{l}\text { Nichtarbeitslose } \\
\text { Leistungsempfänger }\end{array}$ & & \\
\hline
\end{tabular}

1 Restabwicklung für 2005

\section{Zum Nachweis der Wirkung aktiver Arbeitsmarktpolitik}

Maßnahmen aktiver Arbeitsmarktpolitik sind an der Schnittstelle zwischen dem Berufsbildungssystem und dem Arbeitsmarkt angesiedelt. Um hier nachhaltige Veränderungen zu bewirken, bedarf es kohärenter und konsistenter politischer Entscheidungen und Maßnahmen. Ein häufiges Problem ist dabei die Gewährleistung der Konsistenz von Maßnahmen. Fehlende interne Konsistenz kann zu geringer Effektivität und Effizienz führen. Das gilt 6 Quelle: ANBA $(2006,12)$ 
umso mehr, wenn Wechselwirkungen mit anderen Maßnahmen oder Reformen am Arbeitmarkt oder im Berufsbildungssystem ignoriert werden.

Der Einfluss von Koordinationsmechanismen und -systemen, die für die Steuerung aktiver Arbeitsmarktpolitik und deren Umsetzung durch entsprechende Maßnahmen genutzt werden können, variiert mit dem Grad ihrer Konkretion, der Verbindlichkeit von Regeln und Zielen sowie mit dem Grad der Einbindung der entsprechenden Akteure und der öffentlichen Aufmerksamkeit gegenüber der jeweiligen Maßnahme.

Konkrete politische Einflüsse können dabei nur indirekt auf der Basis verschiedener output-orientierter Variablen gemessen werden, die wiederum an die zuvor gesetzten Ziele gekoppelt sein müssen. Dabei reicht eine Übereinstimmung von Maßnahmen und Zielen zur Messung des Impacts nicht aus, sondern es muss zudem nachweisbar sein, dass die Maßnahmen tatsächlich und ausschließlich in Folge politischer Strategien ergriffen wurden. So ist durchaus der Fall denkbar, dass eine Übereinstimmung von Maßnahmen und Richtlinien vorliegt, diese aber in keinem kausalen Zusammenhang stehen, sondern von anderen Faktoren beeinflusst wurden. In dem Nachweis der Relation von Ursache und Wirkung liegt somit eines der Kernprobleme der Impactmessung, die sich bei Maßnahmen aktiver Arbeitsmarktpolitik durch die Frage verschärft, ob der gemessene Erfolg auch ohne die Teilnahme an einer Maßnahme zustande gekommen wäre.

Daneben zeigen sich weitere grundlegende Evaluationsprobleme. So wird der Impact der Maßnahmen anhand der Dauer und Nachhaltigkeit der Eingliederung in den Arbeitsmarkt gemessen (Effektivitäts- bzw. Erfolgskriterium). Bei dem Versuch der Übertragung von Maßnahmen, die diesen Kriterien genügen, ergeben sich dadurch Probleme, dass der betrachtete Zeithorizont, die Zielgruppenfokussierung, das Nebeneinander mehrerer oder konkurrierender Maßnahmen sowie der politische und sozio-kulturelle Kontext nicht direkt übertragbar sind - dies gilt gleichermaßen für die Übertragbarkeit von internationalen Erfahrungen nach Deutschland wie auch für innerdeutsche Transferversuche. Eine weitere Schwierigkeit stellt die Vermischung von Effektivitäts- und Effizienzkriterien dar, zumal fiskalische Effekte einer Maßnahme wie direkte Verwaltungs- oder Implementierungskosten üblicherweise nicht in die Berechnungen einfließen. Paradoxerweise sind meist die ineffizientesten (da teuersten) Maßnahmen zugleich die „besten“, d.h. die effektivsten in dem Sinne, dass sie eine nachhaltige Eingliederung in den ersten Arbeitsmarkt fördern (Sianesi 2002, 126).

Zudem kann die arbeitsmarkt- und sozialpolitisch erwünschte Förderung ,adäquater“ Beteiligungen bestimmter Zielgruppen ALMP-Maßnahmen ebenfalls zu Effektivitäts- und Effizienzverzerrungen führen.

Weiterhin lässt eine Konzentration aufEffektivitätskriterien all jene außerökonomischen Kriterien außer acht, die mit dem Effektivitätskriterium korrelieren, aber schwer zu messen sind: Psychologische Auswirkungen wie gesundheitliches Wohlbefinden oder psychische Stabilität als Folge einer erfolgreichen Teilnahme an ALMP-Maßnahmen erscheinen plausibel, stehen aber weder im Vordergrund der Evaluationen noch sind sie empirisch so zugänglich, dass kausale Wirkungen eindeutig identifizierbar wären. Die Forderung nach einer stärkeren Beachtung der nicht-effektivitätsbezogenen Impacts von ALMP ist damit zwar immer wieder betont worden, spielt aber bei der konkreten Effektivitätsevaluation eine untergeordnete Rolle (Descy/Tessaring 2005; Walsh/Parsons 2004).

Ein weiteres grundlegendes Problem besteht in den Stichprobengrößen und der Teilnehmendenzusammensetzung. Neben den oft geringen Stichprobengrößen spielt die Effektheterogenität eine bedeutende Rolle, d.h. die Unterschiedlichkeit der Effekte je nach 
Teilnehmer bzw. Teilnehmerin, wodurch die Aussagekraft der Durchschnittswerte, die sich bei allen empirischen Evaluationsstudien ergeben, im individuellen Fall zu relativieren ist. So müsste der Erfolg einer Maßnahme - zumindest theoretisch - immer im Vergleich zur Nichtteilnahme an einer Maßnahme im individuellen Fall untersucht werden. Sofern Kontrollgruppen in Untersuchungen einbezogen werden, dürften sich diese im Idealfall lediglich durch die Nichtteilnahme von den Teilnehmendengruppen unterscheiden, was de facto nicht möglich ist und wodurch lediglich mit Durchschnittswerten gerechnet werden kann. Damit kommt es immer zu Selektionsverzerrungen, gleichgültig, ob eine reale kontrafaktische Situation in die Untersuchung einbezogen ist oder ob nur mit hypothetischen Vergleichsgruppen gearbeitet wird.

Letztlich ist eine Differenzierung zwischen makro- und mikroökonomischen Effekten und damit zwischen direkten und indirekten Effekten von ALMP vorzunehmen. Wenngleich viele Studien vorab spezifizieren, auf welcher Ebene Effekte untersucht werden sollen, ist eine strikte Trennung kaum möglich, weil sich ALMP sowohl auf Individuen als auch auf die Arbeitslosen- bzw. Beschäftigungssituation auswirkt:

\section{Tab. 3: Effekte von ALMP ${ }^{7}$}

\begin{tabular}{|c|c|}
\hline $\begin{array}{c}\text { Mikroökonomische Perspektive } \\
\text { (Individuen, direkte Effekte, Bruttoeffekte) }\end{array}$ & $\begin{array}{c}\text { Makroökonomische Perspektive } \\
\text { (Beschäftigungsquote, indirekte } \\
\text { Effekte/Nettoeffekte) }\end{array}$ \\
\hline - Matching-Prozess & - Mitnahmeeffekte \\
\hline - Produktivität der Teilnehmenden & - Substitutionseffekte \\
\hline - Lohnfindungsprozesse & - Verdrängungseffekte \\
\hline - Arbeitsplatzangebot & - Steuereffekte \\
\hline & - Multiplikatoreneffekte \\
\hline
\end{tabular}

Während mikroökonomische Analysen auf den kausalen Bruttoeffekt einer Maßnahme fokussieren, sind gesamtwirtschaftliche Nettoeffekte von ALMP nur durch makro-ökonometrische Verfahren ermittelbar und berücksichtigen die Tatsache, dass eine Maßnahme auch indirekt die Beschäftigungschancen von Nicht-Teilnehmenden beeinflussen kann. Konle-Seidl (2005: 16) weist darauf hin, dass der Nettoeffekt einer Maßnahme gegenüber dem Bruttoeffekt nicht nur geringer, sondern sogar negativ sein kann.

Zusammenfassend lässt sich festhalten, dass vor der Evaluation von ALMP und der Ermittlung ihres Impacts eine Reihe von Zielvorstellungen präzisiert werden müssen, um zu vergleichbaren und robusten Ergebnissen zu gelangen. Dazu gehören:

- Eine Entscheidung zwischen formativer (systembeeinflussender) und summativer (bewertender und begutachtender) Evaluation,

- die Präzisierung der konkreten Outcomes, Veränderungen und Resultate, anhand derer (in Relation zu der Vielzahl möglicher Outcomes) die Wirksamkeit untersucht werden soll,

- die konkreten zu messenden Ziele in Relation zu den meist vage formulierten politischen Zielen und Strategien sowie

- die Ebenen, Mittel und Methoden der Evaluation (Descy/Tessaring 2005, 135; KonleSeidl 2005).

7 In Anlehnung an Calmfors (1994) 


\section{Maßnahmen aktiver Arbeitsmarktpolitik im Überblick}

Ohne die Maßnahmentypen im Einzelnen zu analysieren, soll zunächst ein Überblick über die Arten und die Wirksamkeit der traditionellen und neueren ALMP-Instrumente in Deutschland gegeben werden, bevor auf ALMP im internationalen Vergleich eingegangen wird.

Bei ALMP wird national wie auch international üblicherweise differenziert zwischen

- Beratungs- und Vermittlungstätigkeiten der Arbeitsämter,

- Qualifizierungsmaßnahmen,

- Maßnahmen für Jugendliche,

- beschäftigungsschaffenden Maßnahmen (Einstellungszuschüsse, Schaffung temporärer Beschäftigung, Existenzgründungszuschüsse) sowie

- Maßnahmen für Behinderte. ${ }^{8}$

Umfassende Evaluationen der ALMP-Maßnahmen in Deutschland waren bislang nur eingeschränkt möglich, da im Gegensatz zu anderen europäischen Ländern lange Zeit keine administrativen Datensätze zur Verfügung standen. Erst seit der Einführung des SGB III 1998 und der damit verbundenen gesetzlichen Verpflichtung zur Evaluation werden entsprechende Daten erhoben. Ähnliches gilt für die Daten, die im Zuge der „Hartz“-Evaluation erfasst werden. Hierbei sind vier Datenquellen mit administrativen Informationen auf individueller Ebene von besonderem Interesse, die in der Integrierten Erwerbsbiographie (IEB) des IAB zusammengeführt werden und Aufschluss über die Wirksamkeit von ALMP ermöglichen. Dazu gehören:

- die Maßnahme-Teilnehmer-Gesamtdatenbank (MTG) mit Daten über die individuelle Teilnahme an Maßnahmen und die Rekonstruktion von Maßnahmekarrieren,

- die BA-Geschäftsdaten aus dem Bewerberangebot (BewA),

- die IAB-Leistungsempfängerhistorik $(\mathrm{LeH})$ und

- die IAB-Beschäftigungshistorik (BeH).

$\mathrm{Zu}$ den traditionellen Instrumenten der ALMP gehören die Förderung der beruflichen Weiterbildung, die staatlich geförderten Arbeitsbeschaffungsmaßnahmen (ABM) sowie Lohnsubventionen an Unternehmen bei der Beschäftigung bestimmter Problemgruppen. Durch die Förderung der beruflichen Weiterbildung $(\mathrm{FbW})^{9}$ sollen durch inner- und außerbetriebliche Kurse die (Weiter)Beschäftigungschancen von Arbeitssuchenden gefördert werden.

$\mathrm{Zu}$ den Instrumenten der temporären Schaffung von Arbeitsplätzen gehören zudem Strukturanpassungsmaßnamen (SAM), durch die ebenfalls die Wiederbeschäftigungschancen der Teilnehmenden verbessert werden sollen. SAM und ABM dienen allerdings nicht vorrangig der Förderung der Beschäftigungsfähigkeit der Individuen, sondern sie sind vielmehr sozialpolitische Maßnahmen zur Schaffung von Arbeitsgelegenheiten (KonleSeidl 2005, 6).

Lohnkostensubventionen werden nach SGB III an private Unternehmen in Form von

8 Die von EUROSTAT vorgelegte Kategorisierung umfasst insgesamt eine ausdifferenziertere Kategorisierung, nämlich a) allgemeine Dienste der öffentlichen Arbeitsverwaltungen (PES), b) Intensive Beratung und Unterstützung bei der Arbeitssuche, c) Aus- und Weiterbildung, d) Arbeitsplatztausch und Job-Sharing, e) Beschäftigungsanreize, $f$ ) Eingliederung von Behinderten, g) direkte beschäftigungsschaffende Maßnahmen (ABM, Zusatzjobs), h) Gründungsinitiativen, i) Einkommensunterstützung für Arbeitslose, j) Vorruhestandsmaßnahmen.

9 Nach dem alten Arbeitsförderungsgesetz (AFG) als Fortbildungs- und Umschulungsmaßnahme (FuU) bezeichnet. 
Eingliederungszuschüssen für bestimmte Problemgruppen des Arbeitsmarktes bezahlt, spielen aber mittlerweile in Deutschland eine untergeordnete Rolle (Caliendo/Steiner 2005, 400). In einer Reihe von Modellprojekten wurde zudem mit Arbeitnehmerlohnzuschüssen experimentiert (Einstiegsgeld in Baden-Württemberg, Kombilohn in NRW), deren Ziel in der Verbesserung der Anreize zur Aufnahme einer regulären Beschäftigung bestand.

Im Zuge der Neuorientierung der ALMP durch die „Hartz“-Gesetze wurde eine Reihe von Vorschriften zu bestehenden Maßnahmen vereinheitlicht, so z.B. die Zusammenführung von ABM und SAM; zudem wurden einige Leistungen zeitlich enger befristet und pauschaliert. Eine stärkere Gewichtung erhält seither die Förderung von Jugendlichen mit dem Ziel des Abbaus von Jugendarbeitslosigkeit. Ferner wurden Bildungsgutscheine eingeführt, die unter bestimmten Bedingungen bei einer zertifizierten Weiterbildungseinrichtung freier Wahl eingelöst werden können. Weitere Neuerungen sind

- die Einführung von Personal-Service-Agenturen (PSA) zur Erzielung besserer Vermittlungserfolge,

- Vermittlungsgutscheine für Arbeitssuchende,

- die Beschäftigungsförderung im Bereich geringer Erwerbseinkommen (Mini-Jobs und Midi-Jobs),

- Existenzgründungszuschüsse und

- Lohnersatzleistungen nach dem Prinzip des Förderns und Forderns (Sozialgeld und Arbeitslosengeld II).

Bei den Änderungen der ALMP zeigt sich insgesamt eine deutliche Verschiebung von reinen Förderinstrumenten hin zu solchen, die die Selbstständigkeit von Arbeitssuchenden nicht nur fördern, sondern auch fordern und Erfahrungen am ersten oder zweiten Arbeitsmarkt vermitteln sollen. Dagegen nimmt die Bedeutung der traditionellen ALMP-Instrumente ab.

Bezüglich der Wirksamkeit traditioneller und neuerer ALMP-Instrumente in Deutschland zeigen sich keine einheitlichen Forschungsergebnisse. So beobachten Eichler und Lechner (2002) im Zeitraum von 1992 bis 1997 eine ABM für ostdeutsche Langzeitarbeitslose zwischen 22 und 52 Jahren. Anhand der Beschäftigungsquote innerhalb von und fünf Jahre nach der Maßnahmenteilnahme gelangen die Autoren mit Hilfe eines Matching-Verfahrens (Partial Propensity Score Matching ${ }^{10}$ ) zu dem Ergebnis, dass eine signifikante und nachhaltige Reduktion der Arbeitslosenquote von ostdeutschen Langzeitarbeitslosen durch ABM nur bei Männern erzielt werden konnte, während das Risiko für Frauen nur für kurze Zeit nach der Teilnahme reduziert und zu keiner dauerhaften Situationsverbesserung führte.

Fitzenberger und Speckesser (2005) zeigen für den Beobachtungszeitraum von 19931997 anhand von Wiederbeschäftigungsraten ${ }^{11}$ von Arbeitslosen und von Arbeitslosigkeit Bedrohten - ebenfalls unter Nutzung eines Matching-Verfahrens (Propensity Score Matching) -, dass berufliche Weiterbildung in Westdeutschland kurzfristig zu locking-in-Effekten führt, langfristig dagegen die Beschäftigungsquote signifikant steigt. Für Ostdeutschland war dieser Effekt allerdings nicht zu beobachten.

$\mathrm{Zu}$ widersprüchlichen Ergebnissen gelangen auch Hujer und Wellner (2000) sowie Klose und Bender (2000). Sie zeigen anhand der beruflichen Weiterbildung für Arbeitslose in Ost- und Westdeutschland in den Zeiträumen von 1985-1992 (Hujer/Wellner) und von 1986-1990 (Klose/Bender), dass kurz- und langfristig keine, signifikant positive oder signifikant negative Effekte auf die Beschäftigungswahrscheinlichkeit sowie auf die

10 Differenz-von-Differenzen-Ansatz.

11 Die Beschäftigungsraten wurden in dieser Studie bis zu drei Jahren nach Teilnahmebeginn evaluiert. 
Stabilität der an die Maßnahmen anschließenden Beschäftigungsverhältnisse zu erwarten sind. Somit verdeutlichen sie die unterschiedliche Wirksamkeit von ALMP innerhalb eines einzelnen Maßnahmentypus. Während Hujer und Wellner (2000) für ihre Untersuchung ebenfalls auf das Propensity Score Matching als Verfahren zurückgreifen, nutzen Klose und Bender (2000) ein hierarchisches Matching, bei dem auf der Basis von 985 Teilnehmenden- und Vergleichspersonen aus Fortbildungs- und Umschulungsmaßnahmen (FuU) ereignisanalytische Schätzungen zur Wiederaufnahme von Beschäftigung durchgeführt werden. Sie kommen zu dem Schluss, dass ,der kurzfristige (schwache) positive Effekt einer FuU-Maßnahme auf die Wiederbeschäftigung in längerfristiger Perspektive nicht gestützt [wird]. Eine FuU-Maßnahme trägt darüber hinaus auch nicht dazu bei, dass das erreichte Beschäftigungsverhältnis dauerhafter als das der gewählten Vergleichsgruppe ist. Ehemalige Teilnehmer beenden das erste Beschäftigungsverhältnis in der Nachgeschichte sogar signifikant schneller als die zum Vergleich herangezogenen Nicht-Teilnehmer, um dann erneut in Leistungsbezug einzumünden“ (Klose/Bender 2000, 439).

Caliendo/Hujer/Thomsen (2006) nutzen für ihre Analyse der Wirkungen von ABM einen Datensatz mit Informationen über alle Teilnehmer einer ABM-Maßnahme (Beginn Februar 2000) sowie über alle Teilnehmer einer Kontrollgruppe von Nichtteilnehmern. ${ }^{12}$ Die Besonderheit dieses Datensatzes liegt darin, dass er erstmals die Untersuchung der Aufnahme regulärer Beschäftigung ermöglicht. Zudem werden auch in dieser Studie Matching-Verfahren genutzt, um die Maßnahmeeffekte auch mit Blick auf regionale Differenzen und besondere Zielgruppen am Arbeitsmarkt differenzieren zu können.

Berücksichtigt man die beschränkte Aussagekraft des Datenmaterials, das bis zur Einführung der Evaluationspflicht vorlag sowie die zeitliche Spanne, die zwischen Datenerhebung und der Verfügbarkeit der Daten entsteht, ergibt sich für die Wirksamkeit ${ }^{13}$ von ALMP insgesamt folgendes Bild:

- ABM weist eher negative durchschnittliche Effekte auf die individuellen (Wieder)Beschäftigungschancen von Arbeitssuchenden auf.

- FbW weist keine durchschnittliche Steigerung der Beschäftigungschancen auf. Sofern diese überhaupt zu erkennen sind, beziehen sie sich auf außerbetrieblich durchgeführte und kurzfristige FbW.

- Während der Teilnahme an einer ABM oder FbW sinkt die Beschäftigungswahrscheinlichkeit.

- Nach Beendigung einer Maßnahme haben die Teilnehmenden eine geringere Beschäftigungswahrscheinlichkeit als die Individuen der Kontrollgruppe. Diese geringere Beschäftigungswahrscheinlichkeit sinkt, wenn überhaupt, erst langfristig.

Eine deutliche Ausnahme weisen hier die für Westdeutschland gültigen Untersuchungsergebnisse von Fitzenberger und Speckesser (2005) auf.

Während die Ergebnisse mikroökonomischer Studien eher uneindeutig sind, lassen sich aus makroökonomischen Studien für Deutschland zwei Aspekte zweifelsfrei ableiten, nämlich dass ALMP im Allgemeinen einen lohnmoderierenden Effekt haben und dass sie das Beschäftigungsniveau erhöhen (Kraft 1998).

12 Diese waren im gleichen Zeitraum arbeitslos.

13 Hier im Sinne der (Wieder)Eingliederung in den Arbeitsmarkt. 


\section{Impacts von ALMP im internationalen Vergleich}

Im internationalen Kontext liegt ebenfalls eine Reihe von Studien über die Wirksamkeit von ALMP vor. Dabei zeigt sich, dass Aussagen über den Einfluss von ALMP nur in Abhängigkeit von Land, Region, Zielgruppe und Systembedingungen getroffen werden können (Hujer et al. 2004; Fischer/Tholoriat 2006; Kluve 2006; Martin 1998). Ergebnisse, die auf eine prinzipielle Wirksamkeit bestimmter Maßnahmetypen schließen ließen, liegen auch hier nicht vor.

So zeigen Panelregressionen, bei denen die Effekte zusätzlicher Variablen wie Kündigungsschutzbestimmungen, die Art der Lohnfindungsprozesse oder die Höhe und Dauer von Lohnersatzleistungen in die Berechnung einfließen, dass ALMP entweder keinen signifikanten oder nur einen sehr mäßig positiven Einfluss auf die nachhaltige Verbesserung der Beschäftigungsquote hat. Eindeutig ist dagegen, dass großzügige Transferleistungen und strikt konzipierte Maßnahmen, bei denen z.B. der Arbeitswillen regelmäßig getestet wird, wesentlich besser und nachhaltiger zur Reduktion des Arbeitslosigkeitsniveaus führen als großzügige Transferleistungen mit niedrigen oder fehlenden Auflagen (Nickell/van Ours 2000).

In europäischen Studien werden darüber hinaus vorwiegend Verweildauermodelle und Matching-Methoden genutzt, um die Effekte von ALMP zu untersuchen. Die Wahl dieser Modelle und Methoden liegt darin begründet, dass die EU-Beschäftigungspolitik lange Zeit stärker die Reduktion der Arbeitslosigkeit als die Erhöhung individueller Beschäftigungschancen fokussierte. Dennoch zeigen sich auch hier unabhängig von der Wahl der Modelle und Methoden widersprüchliche Ergebnisse. So hat Martin (1998) gleichermaßen lohnmoderierende Effekte als auch eine Förderung des Mismatch am Arbeitsmarkt nachgewiesen. Bei Frauen fallen diese Effekte zudem wesentlich signifikanter aus als bei Männern, was vermutlich mit der Gesamtpartizipationsrate von Frauen am Arbeitsmarkt insgesamt und speziell im Zusammenhang mit Vollbeschäftigung zu sehen ist.

Neuere mikroökonomische Studien in verschiedenen europäischen Ländern zeigen ebenfalls widersprüchliche Ergebnisse. So weist Arellano (2005) positive Effekte beruflicher Bildung für weibliche Arbeitssuchende in Spanien nach, indem er die Effekte der spanischen Arbeitsmarktreform von 2001 untersucht. Durch diese Reform sollten vor allem neue Arbeitsplätze geschaffen werden bzw. bestehende erhalten bleiben. Auf der Basis eines Datensatzes von Arbeitslosen im Zeitraum von Januar 1997 bis September 2003 und unter Nutzung von Schätzverfahren ${ }^{14}$ weist Arellano zudem nach, dass die Reform für Männer unter 30 Jahren negative Auswirkungen hat, während in der Gruppe der über-55-jährigen Männer geringe positive Effekte zu finden sind.

Hämäläinen und Ollikainen (2004) zeigen eine signifikante Verbesserung der Beschäftigungswahrscheinlichkeit und des Lohnniveaus nach erfolgreicher Teilnahme an beruflicher Bildung auf. Dabei analysieren sie die Langzeiteffekte der finnischen ALMP für junge Arbeitslose anhand verschiedener Indikatoren (u.a. Programmteilnahme, Beschäftigungsquote, Ausbildungsniveau). Die Maßnahmeeffekte werden durch ein Matching-Verfahren (non-parametric propensity score matching) analysiert und weisen die Tendenz auf, dass die Förderung beruflicher Bildung sowohl die Beschäftigungschancen als auch die Einkommenshöhe verbessert.

14 Basierend auf einem mixed proportional hazard rate model, nicht-experimentelles Design. 
Mit dem Youth Unemployment Programme (YUP) hat Dänemark 1996 eine radikale Arbeitsmarktreform zur Verringerung der Arbeitslosenquote von gering qualifizierten jungen Menschen durch speziell für diese Zielgruppe zugeschnittene Berufsbildungsmaßnahmen initiiert. Eine Studie von Jensen/Rosholm/Svarer (2003) zeigt anhand von Teilnehmendenübergangsquoten einen positiven Effekt des Programms, der hauptsächlich durch drei Items hervorgerufen wird, i.e. das Programm, Sanktionen unterschiedlicher Art und den Announcement-Effekt. ${ }^{15}$ Die Ergebnisse weisen keine signifikanten Effekte für die Dauer der Arbeitslosigkeit aus, zeigen aber, dass sich durch die Maßnahmenteilnahme die Übergangsquote in weiterführende Schulen erhöht.

Cockx (2003) untersucht die Wirkung einer Berufsbildungsmaßnahme für belgische Arbeitslose im Zeitraum von 1989-1993 mit Hilfe eines Schätzverfahrens. Die Ergebnisse zeigen, dass die Übergangsrate in Beschäftigung während der Teilnahme sinkt und nach Teilnahmeende deutlich steigt (von $42 \%$ auf $73 \%$ ). Dieser Effekt setzt allerdings frühestens vier Monate nach Maßnahmenende ein.

Diese Ergebnisse verdeutlichen, dass es keine Maßnahme gibt, die für alle Zielgruppen und in allen Ländern erfolgreich ist. Auch konjunkturelle Einflüsse, die Arbeitsmarkt- und politische Lage haben erhebliche Auswirkungen auf die Impacts berufsbildungsbezogener ALMP. So haben sich z.B. erhebliche Effektivitätsdifferenzen von ALMP in Ost- und Westdeutschland gezeigt, die auf einer fehlenden Zielgruppenorientierung basieren und sich in locking-in-Effekten während der Maßnahmenteilnahme äußern (Caliendo/Hujer/ Thomsen 2005). ${ }^{16}$

Darüber hinaus zeigen die Untersuchungen zwar deutlich, dass sich eine Verbindung von Kontrolle und Unterstützung, orientiert an dem Motto „Fördern und Fordern“, als am wirksamsten erweist. Dennoch lässt dieses Ergebnis keine Rückschlüsse auf die konkrete Ausgestaltung der ALMP-Maßnahmen zu. Als weit schwieriger als die Frage nach der Effektivität (im Sinne der Eingliederung in den Arbeitsmarkt) erweist sich jene nach dem Mehrwert bzw. nach all jenen positiven Effekten von ALMP, die sich nicht direkt in der Aufnahme oder dem Beibehalt einer Beschäftigung ausdrücken, sondern zu den indirekten Effekten aus mikroökonomischer Perspektive gehören. Die Schwierigkeit der Bewertung dieser Effekte liegt in erster Linie darin, dass sich diese Impacts weitgehend quantitativen und standardisierten Evaluationsmethoden entziehen, gleichwohl ihre Existenz unumstritten ist. Unklar bleibt zudem die Auswirkung von ALMP auf die Verringerung des Fachkräftemangels, der allerdings mit Blick auf die stetig wachsenden Anforderungen am Arbeitsmarkt nicht nur durch die Qualifizierung von Arbeitssuchenden, sondern nur durch dauerhafte Qualifizierung aller Arbeitskräfte abgefedert werden kann (Steedman 2005).

\section{Grundprinzipien der Gestaltung erfolgreicher ALMP}

Aus den vorliegenden Studien lassen sich eine Reihe grundlegender Forderungen und Ergebnisse zur Gestaltung von ALMP ableiten, die zwar keine Erfolgsgarantie, aber die dafür notwendige Grundlage darstellen.

15 Beeinflussung der Erwartungen und des tatsächlichen Verhaltens der Akteure durch die Ankündigung wirtschaftspolitischer Maßnahmen.

16 Locking-in-Effekte bezeichnen die starke Einschränkung der Suchanstrengungen im Hinblick auf eine Beschäftigung von Teilnehmenden an Maßnahmen während des Teilnahmezeitraums. 


\section{Vorkenntnisse und Arbeitsmarktbezug}

Dazu gehört zunächst eine Erweiterung vorhandener Kenntnisse, Fähigkeiten und Fertigkeiten, d.h. das Anknüpfen an das Vorwissen der Teilnehmenden, wodurch wiederum ihr (Selbst)Vertrauen gestärkt wird. Umgekehrt kann eine fehlende Anknüpfung an vorhandenes Wissen in Demotivierung und Teilnahmeabbruch münden. Ist die Anforderung an die Teilnehmenden dagegen zu hoch, ist die Investition in die Maßnahme zugleich eine Fehlinvestition. Ferner zeigt sich, dass viele ALMP-Maßnahmen zeitlich zu kurz angelegt sind, um nachhaltige Erfolge zu erzielen, was vor allem für die Maßnahmen gilt, die auf die Förderung der Lese- und Rechenfähigkeiten ausgerichtet sind. Zugleich bietet die Fokussierung von grundlegenden Fähigkeiten eine Möglichkeit der Förderung von lernerzentrierten Strategien und Praktiken, insbesondere durch die Nutzung neuer Medien.

Berufliche Weiterbildung für Arbeitssuchende muss außerdem klare Verbindungen zu den Anforderungen am Arbeitsmarkt aufweisen und nicht nur auf grundlegende Fertigkeiten und formale Qualifikationen zielen. Dies setzt wiederum eine Verbesserung der Beratung und Begleitung von Arbeitssuchenden voraus: So erweisen sich individuell abgestimmte Pläne zur Reintegration in den Arbeitsmarkt als erfolgreich, da sie einen realistischen Weg zum Erhalt einer Arbeitsstelle und zur Aufrechterhaltung einer Beschäftigung aufzeigen. In solchen Konzepten ist die berufliche Bildung allerdings nur ein Faktor unter mehreren, in deren Vordergrund eine eigenverantwortliche Lebensführung steht (Brandsma, 2001).

\section{Teilnehmendenstruktur}

Bei der Analyse der Teilnehmerstruktur von Weiterbildungsmaßnahmen für Arbeitslose und Personen mit Arbeitslosigkeitsrisiko lässt sich eine Überrepräsentation von männlichen, höher qualifizierten, ledigen, kinderlosen, jüngeren und kurzzeitig Arbeitslosen finden. Diese Überrepräsentation lässt sich nicht nur bei Weiterbildungsprogrammen für arbeitslose Erwachsene im Allgemeinen finden, sondern kann auch innerhalb von Programmen für bestimmte Zielgruppen innerhalb der Gruppe der Arbeitslosen beobachtet werden (wie z.B. ältere Arbeitslose oder Langzeitarbeitslose).

Die Vielfalt der ungesicherten, nur teilweise überprüften oder nur für spezifische Gruppen, Regionen und Zeiträume gültigen Ergebnisse ist in Relation zu den gesicherten Ergebnissen erheblich größer. Dazu gehören z.B. der Einfluss von Motivation auf die Teilnahmebereitschaft, die Strategien der (lebenslangen) Beratung und Begleitung sowie die Kooperation zwischen Akteuren und die Effektivität der Angebote.

Im Hinblick auf die Teilnahmemotivation haben verschiedene Studien gezeigt, dass sich eine Reihe von Faktoren für Arbeitssuchende bzw. Arbeitslose als Teilnahmenerschwernis erweist: Die Teilnahmebereitschaft ist umso niedriger, je unqualifizierter bzw. fehlqualifizierter die Arbeitslosen sind und je stärker strukturelle Benachteiligungen kumulieren (Brandsma 2001). Mehrere Gründe scheinen dafür verantwortlich zu sein:

- Die Unsicherheit über mögliche Vorteile einer Teilnahme;

Versagensängste, ein negatives Selbstbild und die Angst vor Exklusion;

fehlende Verbindungen zwischen den Inhalten des Angebots und den tatsächlichen Bildungsbedürfnissen der Arbeitssuchenden;

eine innere Einstellung, bei der prinzipiell dem Arbeiten bzw. einer beruflichen Tätigkeit Vorrang vor Lernaktivitäten und Qualifizierungsmöglichkeiten gegeben wird sowie

- fehlende Orientierung an den Bedürfnissen der Individuen: So ist offensichtlich, dass 
z.B. ältere männliche Langzeitarbeitslose andere Qualifizierungsbedürfnisse haben als jüngere weibliche Kurzzeitarbeitslose.

Darüber hinaus kann die Beratung und Betreuung von Arbeitslosen und Arbeitssuchenden die Motivation der Betroffenen zur Teilnahme an beruflicher Bildung positiv oder negativ beeinflussen. Das wichtigste Konzept besteht darin, nicht nur berufliche Bildung anzubieten, sondern auch eine entsprechende Arbeitshaltung zu vermitteln, was eine häufig unterschätzte Aufgabe darstellt. Vranken und Frans $(2001,142)$ haben in diesem Zusammenhang darauf hingewiesen, dass Arbeitslose oft mehr Zeit benötigen, um sich an einfache Aufgaben wie "sticking to time schedules, meeting strict requirements, integration into a team, accepting authority, and concentrating on one task for a longer time" als fachliche Kompetenzen zu erwerben zu gewöhnen.

\section{Kooperation und Kontrolle der Akteure}

Für eine effektive Kooperation sollte der Fokus darauf liegen, Koordinationsschemata und Netzwerke zwischen Arbeitsmarktakteuren auf regionalem, nationalem und supranationalem Level zu etablieren und neue Arbeitsmarktarrangements zu schaffen. Werden dabei Netzwerke als Koordinationsformen genutzt, bewirken hierarchische Kooperationsformen verlässlichere Möglichkeiten der Kooperation und der Implementation von neuen Strategien als andere Arten der Kooperation. Feste Bestandteile der Kooperation müssen auf Grundlage der Forschungsergebnisse zudem kontinuierliche Informationsaustausche sowie ein langfristig ausgerichtetes und zuverlässiges Monitoring von beruflichen Bildungsangeboten sein, mit deren Hilfe die Qualität und die direkte Einbindung der Sozialpartner sowie der Anbieter von Maßnahmen durchgeführt werden kann.

Dennoch bleibt damit ein grundlegendes Problem ungelöst: Die meisten Bildungsmaßnahmen für die genannten Zielgruppen werden von unabhängigen Bildungsträgern angeboten, denen häufig eine aktuelle Arbeitsmarktorientierung fehlt. Daher sollte die Stärkung der Kooperation und Koordination nicht nur eine bessere Zusammenarbeit von Politik und Bildungsträgern, sondern auch jene zwischen Unternehmen, Wissenschaft, Politik und Anbietern fördern.

\section{$6 \quad$ Resümee und Ausblick}

Vor allem ältere Evaluationsstudien haben eher negative Effekte von traditionellen Instrumenten der ALMP nachgewiesen. Das mag an methodischen Schwächen der Untersuchungsdesigns liegen, aber auch - gerade im Fall Deutschlands - an unzureichenden Stichproben und einer daraus resultierenden begrenzten Aussagekraft von Ergebnissen, die vorwiegend auf Durchschnittswerten beruhen und keine Differenzierung nach Teilnehmendengruppen ermöglichen. Dennoch geben auch neuere Untersuchungen im nationalen wie im internationalen Kontext trotz der Behebung empirischer Schwächen und der Beachtung der Teilnehmendenheterogenität keinen Anlass für eine grundlegend optimistische Haltung. So liegen nur wenige Untersuchungen vor (Speckesser/ Fitzenberger 2005), die eine langfristige Verbesserung der Beschäftigungschancen trotz vorübergehender locking-in-Effekte während der Teilnahme an ALMP-Maßnahmen aufweisen. Die Gründe dafür, weshalb welche Maßnahme für eine bestimmte Teilnehmendengruppe erfolgreich ist oder eben nicht, bleibt dabei weitgehend unklar: „Während die Ergebnisse der meisten 
statistischen Evaluationsstudien darauf hinweisen, dass durch die AAMP im Durchschnitt oft keine signifikanten oder sogar negative Beschäftigungseffekte resultieren, sind die Ursachen dafür bisher kaum erforscht. Die vorliegenden Studien schätzen den Erfolg einer bestimmten Maßnahme der AAMP auf der Basis statistischer Modelle ohne explizite Formulierung ökonomischer Verhaltenshypothesen. Dabei lassen sich Hypothesen bezüglich der Wirkungen der mit der Teilnahme an einer bestimmten Maßnahme verbundenen ökonomischen Anreize meist nicht direkt testen. Im günstigsten Fall ergeben sich aus diesen Studien indirekte Hinweise auf mögliche Ursachen für die ermittelten Wirkungen der Maßnahmen“" (Caliendo/Steiner 2005, 415).

Die Identifizierung von erfolgreichen Maßnahmen und die Möglichkeit einer exakteren Berechnung der Effekte für spezifische Teilnehmendengruppen wären jedoch notwendig, um künftige Politikempfehlungen abzuleiten und die Konzeption der Maßnahmen nachhaltig zu verbessern.

Weitgehend unklar bleiben zudem die indirekten Effekten von ALMP, also z.B. Mitnahme- oder Verdrängungseffekte, da sich bisherige Studien auf Effektivitätsanalysen im Sinne einer raschen (Wieder)Eingliederung in den Arbeitsmarkt konzentrieren und nichteffektivititätsbezogene Impacts sowie indirekte Effekte weitgehend unbeachtet bleiben.

Unklar bleibt zudem, welche Auswirkungen ALMP auf die Reduktion des Fachkräftemangels haben. Dies gilt umso mehr, als der Fachkräfte- (und teilweise auch Arbeitskräfte)Mangel längst nicht von allen Arbeitgebern als aktuelles Problem betrachtet wird (Strietska-Ilina 2005) und die Verbindung von Impactforschung und der Ermittlung künftiger Qualifikationserfordernisse erst langsam aufkeimt (Schmidt u.a. 2005).

Für künftige Evaluationsstudien wären bei dem Einsatz individueller Fallbetreuung mit passgenauen Handlungsprogrammen Forschungsansätze nötig, die die Interaktion von Ansprüchen und Zumutbarkeit zur Aufnahme von Arbeit stärker untersuchen und dabei die Individualität der Fälle berücksichtigen. Im Fokus sollte dabei nicht nur die Ausgestaltung künftiger Arbeitsmarktpolitik stehen, sondern vor allem die Entwicklung von Human- und Sozialkapital.

\section{Literatur}

ANBA- Amtliche Nachrichten der Bundesagentur für Arbeit (2006): Arbeitsstatistik 2005 - Jahreszahlen. 54, Sondernummer. Nürnberg

Arellano, F. Alfonso (2005): Evaluating the Effects of labour market reforms. At the margin of unemployment and employment stability: The Spanish case. Economics Working Papers, Universidad Carlos III, Madrid

Brandsma, Jittie (2001): Training and employment perspectives for lower qualified people; in: Pascaline Descy, Manfred Tessaring (Hg.): Training in Europe. Luxembourg, 173-206

Caliendo, Marco, Viktor Steiner (2005): Aktive Arbeitsmarktpolitik in Deutschland: Bestandsaufnahme und Bewertung der mikroökonomischen Evaluationsergebnisse; in: Zeitschrift für Arbeitsmarktforschung, 38, 2/3, 396-418

Caliendo, Marco, Reinhard Hujer, Stephan L. Thomsen (2005): The Employment Effects of Job Creation Schemes in Germany - A Microeconometric Evaluation. IZA Discussion Paper, No. 1512. Bonn

Calmfors, Lars (1994): Active labour market policy and unemployment - A framework for the Analysis of Crucial Design Features; in: OECD Economic Studies, 22, 7-47 
Cockx, Bart (2003): Vocational training of unemployed workers in Belgium. Bonn

Descy, Pascaline, Manfred Tessaring (2005): The value of learning: evaluation and impact of education and training. Third report on vocational training research in Europe. Synthesis report. Luxembourg

Eichler, Martin, Michael Lechner (2002): An evaluation of public employment programmes in the East German State of Sachsen-Anhalt; in: Labour Economics, 9, 143-186

EUROSTAT (2007): European social statistics. Social protection. Expenditure and receipts 19962004. Luxembourg

Fischer, Georg, Luc Tholoniat (2006): The European Social Model and Eastern enlargement; in: Zeitschrift für Arbeitsmarktforschung, 39, 1, 123-142

Fitzenberger, Bernd, Stefan Speckesser (2002): Weiterbildungsmaßnahmen in Ostdeutschland: Ein Misserfolg der Arbeitsmarktpolitik? ZEW Discussion Paper No. 02-16, Mannheim

Hämäläinen, Kari, Virve Ollikainen (2004): Differential effects of active labour market programmes in the early stages of young people's unemployment. VATT Research Reports 115, Helsinki

Hujer, Reinhard, Marco Caliendo (2001): Evaluation of active labor market policy - Methodological concepts and empirical estimates; in: Irene Becker, Notburga Ott, Gabriele Rolf (Hg.): Soziale Sicherung in einer dynamischen Gesellschaft. Frankfurt a. M., 163-199

Hujer, Reinhard, Marco Caliendo, Dubravko Radic (2004): Methods and limitations of evaluation and impact research; in: Pascaline Descy, Manfred Tessaring (Hg.): The foundations of evaluation and impact research. Luxembourg, 131-190

Hujer, Reinhard, Marc Wellner (2000): Berufliche Weiterbildung und individuelle Arbeitslosigkeitsdauer in West- und Ostdeutschland; in: Mitteilungen aus der Arbeitsmarkt- und Berufsforschung, $3,405-420$

Jensen, Peter, Michael Svarer, Michael Rosholm (1999): The Effects of Benefits, Incentives, and Sanctions on Youth Unemployment. Working Paper 99-05, Center for Labour Markets and Social Research, Aarhus

Klose, Christoph, Stefan Bender (2000): Berufliche Weiterbildung für Arbeitslose - ein Weg zurück in Beschäftigung?; in: Mitteilungen aus der Arbeitsmarkt- und Berufsforschung, 33, 3, 421-444

Kluve, Jens (2006): The effectiveness of European active labour market policy. Discussion Paper No. 2018. Bonn

Konle-Seidl, Regina (2005): Lessons learned. Internationale Evaluierungsergebnisse zu Wirkungen aktiver und aktivierender Arbeitsmarktpolitik. IAB Forschungsbericht Nr. 9/2005. Nürnberg

Kraft, Kornelius (1998): An Evaluation of Active and Passive Labour Market Policy; in: Applied Economics, 30, 6, 783-793

Martin, John P. (1998): What works among active labour market policies: Evidence from OECD countries' experiences. Paris

Nickell, Stephen J., Jan van Ours (2000): The Netherlands and the United Kingdom: a European Unemployment Miracle?; in: Economic Policy, 15, 30, 136-180

Schmidt, Susanne L., Olga Strietska-Ilina, Manfred Tessaring, Bernd Dworschak (2005): Ermittlung künftiger Qualifikationserfordernisse. Luxembourg

Sianesi, Barbara (2002): Essays on the evaluation of social programmes and education qualifications. Stockholm

Steedman, Hilary (2005): Neue Herausforderungen für die „Risikogruppe“ am Arbeitsmarkt; in: Susanne L. Schmidt, Olga Strietska-Ilina, Manfred Tessaring, Bernd Dworschak (Hg.): Ermittlung künftiger Qualifikationserfordernisse. Luxembourg, 213-221

Strietska-Ilina, Olga (2005): Untersuchung von Kompetenzknappheit durch eine kombinierte Methode; in: Susanne L. Schmidt, Olga Strietska-Ilina, Manfred Tessaring, Bernd Dworschak (Hg.): Ermittlung künftiger Qualifikationserfordernisse. Luxembourg, 83-95

Vranken, Jan, Mieke Frans (2004): Selection, social exclusion and training offers for target groups; in: Pascaline Descy, Manfred Tessaring (Hg.): Training in Europe. Second report on vocational training research in Europe 2000. Luxembourg, 137-171 
Walsh, Kenneth, Parsons, D. J. (2004): Active policies and measures: impact on integration and reintegration in the labour market and social life; in: Pascaline Descy, Manfred Tessaring (Hg.): The impact of education and training. Third report on vocational training research in Europe. Luxembourg 215-259

Anschrift der Verfasserin:

Prof. Dr. Sandra Bohlinger

Technischen Universität Berlin

Institut für Berufliche Bildung und Arbeitslehre

Franklinstr. 28/29, Sekr. FR4-4

D-10587 Berlin

eMail: sandra.bohlinger@tu-berlin.de

Schlagwörter: Arbeitsmarkt, national/international, Qualifikation/ Aus- und Weiterbildung 